\title{
Treatment of Helicobacter pylori: future therapeutic and prophylactic perspectives
}

\author{
A T R Axon
}

\begin{abstract}
Summary
Helicobacter is one of the most widespread, chronic infections in the world and causes a serious disease with a significant mortality. The organism is difficult to eradicate using antibiotic therapy and to date no vaccine is available for use in humans. The most successful treatments comprise acid suppression in combination with two antibiotics and a series of seven day courses will reliably cure around $85 \%$ of infected individuals. Further modifications of these regimens via the introduction of newer and more effective antibiotics and acid suppressants may enable the treatment to be simplified, made more effective and cause fewer side effects. However, the major challenge is to develop a specific monotherapy targeted against Helicobacter pylori. The $H$ pylori genome has now been sequenced and this provides an opportunity both to identify specific targets for drug therapy, and to facilitate the identification and production of antigens that may be helpful in manufacturing a vaccine. This paper discusses the future of helicobacter therapy and vaccination.
\end{abstract}

\section{Introduction}

Helicobacter pylori gastroduodenitis is a chronic infectious disease, but differs from many others in that once established the organism persists usually for the whole natural life of its host. Unlike syphilis, tuberculosis and many other chronic infections it does not have a dormant period, but causes continuous inflammation. The inflammatory response possesses not only the characteristics of a chronic infection, but is associated with the infiltration of acute inflammatory cells, implying that the inflammatory process actively attacks the host for many years. This unusual parasite/host relationship explains perhaps why difficulties have been experienced in identifying a satisfactory treatment. In most infections antibiotic therapy has merely to tip the balance in favour of the host to enable it to eliminate the parasite. Where $H$ pylori gastritis is concerned, however, the powerful and continued immunological reaction evoked is evaded or modified by the organism in such a way as to render it impotent or it may perhaps even benefit the parasite. It follows that a successful chemotherapy must be capable of eradicating the organism completely as it will receive little assistance from the host immune mechanisms.

In theory therefore it will also be difficult to develop a vaccine because the host already generates humoral and tissue responses to the organism which are demonstrably ineffective.

It is interesting therefore that in spite of these handicaps not only are certain chemotherapeu- tic combinations highly effective, but vaccines have been produced that protect the host in animal models.

The purpose of this paper is to provide an appraisal of the most effective, current treatments available for $H$ pylori, and to speculate on the potential for newer approaches in treatment and prophylaxis.

\section{Antibiotic combinations}

It was recognised at an early stage that although $H$ pylori is sensitive to a wide variety of antibiotics in vitro, when used in vivo none of them worked. As a result most of the treatments currently in use have been developed not by a planned approach, but as a result of empirical experimentation. The main components of modern treatments comprise amoxycillin, macrolides, nitroimidazoles, tetracycline, bismuth salts, and acid suppressants. None are effective when used singly and dual therapy also gives disappointing results. It is only when combinations of three or more drugs are given that acceptable eradication rates are achieved. Furthermore, it is not possible to obtain good results by linking together any three of the medications, the specific combination is important too. As a result of clinical experimentation we now recognise that a number of combinations can be given in a twice daily dose for one week with reasonable expectation of achieving success in $85-95 \%$ of patients who take the whole course. There is therefore a bench mark against which newer drugs or combinations of drugs can be compared.

\section{Proton pump inhibitor based triple therapies}

The most widely used combinations today are triple therapies comprising a proton pump inhibitor, amoxycillin and a macrolide, or alternatively, a proton pump inhibitor, a nitroimidazole (usually metronidazole) and a macrolide. ${ }^{1}$ The macrolide most commonly used in these combinations is clarithromycin which is much more effective than erythromycin because it is concentrated within the gastric mucosa and has a degree of acid resistance. Similar results have been obtained with azithromycin which may hold an advantage in having a long tissue half life and may perhaps enable the length of treatment to be reduced ${ }^{2}$ and improve compliance. The third combination comprises a proton pump inhibitor, amoxycillin and metronidazole. ${ }^{3}$ Recent studies suggest that this particular combination is not quite as effective as the other two. The most cost efficient of these combinations is omeprazole $20 \mathrm{mg}$ (or another proton pump
The General Infirmary Great George Street, Leeds LS1 3EX, UK A Axon 
inhibitor) once daily, metronidazole $400 \mathrm{mg}$ twice daily and clarithromycin $250 \mathrm{mg}$ twice daily for one week. The second combination lansoprazole $30 \mathrm{mg}$ (or another proton pump inhibitor) twice daily, clarithromycin $500 \mathrm{mg}$ twice daily and amoxycillin $1 \mathrm{~g}$ twice daily for one week is more expensive because it requires a higher dose of the proton pump inhibitor and clarithromycin.

It is perhaps surprising that the combinations including metronidazole are so effective bearing in mind the high incidence of metronidazole resistance within most populations. ${ }^{4}$ It seems, however, that when a proton pump inhibitor and clarithromycin are present in the combination it partially negates the effect of metronidazole resistance, rendering organisms that are resistant to metronidazole susceptible. ${ }^{5}$ How exactly this happens is unclear, but it seems that the metronidazole resistance in $H$ pylori is relative rather than absolute ${ }^{6}$ and may be influenced by the $\mathrm{pH}$ and oxygen tension in the microenvironment. The omeprazole, amoxycillin and metronidazole combination may be less effective because it is less successful with metronidazole resistant organisms.

\section{Bismuth combinations}

Bismuth salts were the first medications to be used in the treatment of $H$ pylori though arsenicals were used 70 years ago in an animal model to treat $\mathrm{H}$ felis. ${ }^{7}$ More recently zinc has also been shown to have a useful therapeutic effect as well. ${ }^{8}$ On their own metallic salts are ineffective, but when used in combination with tetracycline, metronidazole and a proton pump inhibitor this quadruple therapy given for a week achieves good results even in patients harbouring metronidazole resistant organisms. ${ }^{9}$ This quadruple therapy can be used as a second line treatment in patients who have failed with the triple therapies described in the previous paragraph, though once a patient has failed with an eradication therapy the likelihood of success second time around is less even when using a different combination.

A new development of bismuth therapy has been the production of ranitidine bismuth citrate (RBC). This molecule combines both bismuth and ranitidine giving the advantage of both bismuth and acid suppression. When used on its own it has no anti-helicobacter effect in vivo. When combined with clarithromycin 500 $\mathrm{mg}$ and given in a dosage of $400 \mathrm{mg}$ twice daily for two weeks, results have been achieved around $90 \% .^{10}$ It seems likely that this combination is also effective when given for one week ${ }^{11}$ though studies using this dosage are limited in number. When RBC is used with two other antibiotics - that is to say metronidazole and clarithromycin or tetracycline and clarithromycin-similar results have been obtained, ${ }^{12}$ but again the number of studies undertaken using these combinations are limited.

Potential modification to current regimes It is possible that some development will still take place with the combinations indicated earlier or indeed new combinations may be shown to be more effective. It is possible that more effective acid reducing agents may become available or quicker acting ones such as a reversible proton pump inhibitor. The latter might have the advantage of working more rapidly and perhaps enabling the length of treatment to be reduced. Longer acting acid suppressants, macrolides and nitroimidazoles might reduce the length of medication courses.

One interesting new drug that has been trialed is nitazoxanide. ${ }^{13}$ This is a nitroimidazole to which $H$ pylori does not seem to develop resistance. To date it has been used in only small numbers of patients.

The combinations could be further improved by producing single capsules containing a mixture of drugs thereby reducing the number of tablets that need to be taken and perhaps improving compliance. However, the committees responsible for registering new pharmaceuticals are, in general, reluctant to accept combinations of more than one drug in a single capsule. An alternative approach would be to link two drugs together in one molecule as with RBC. Newer drugs are being investigated by the pharmaceutical industry, but the most exciting development from an intellectual as well as from a practical point of view is the potential to produce designer drugs based on the information which is now available from the decoding of the helicobacter genome.

\section{Use of the $H$ pylori genome for developing designer drugs}

The genome sequence of $H$ pylori has now been published in full by scientists at The Institute for Genome Research, and has been available, in part, to certain organisations for a longer period. ${ }^{14} \mathrm{H}$ pylori is a relatively simple organism with a small genome of circa $1.7 \times 10^{6}$ nucleotides, varying slightly according to the strain of the organism, and encoding around 1600 genes. Of these, depending on the stringency of the comparison, roughly 55\% have homologs of putative known identity in other organisms, while another $10 \%$ have database homologs of unknown identity. The remaining $35 \%$ are unique to $H$ pylori at this time. This complement of 1600-1700 genes contains a relatively small number which encode proteins whose functions are essential to the viability of the bacterium.

The principle underlying genome based drug development is to identify those essential proteins which are specific to $H$ pylori, and then to isolate, identify and synthesise a small molecule chemical which inhibits the essential activity of such proteins. This approach is designed both to identify a drug that will work as a monotherapy, which would be specific for $H$ pylori and would not interfere with other organisms.

Identification essentiality involves a process whereby attempts are made to mutate a gene and so "knockout" its function. If a gene cannot be mutated, the encoded protein is likely to be essential to the bacterium, and represents a potential target for a therapeutic drug. Many essential proteins are enzymes, making it possible to establish in vitro assays to detect the 
ability of compounds to interfere with the target enzymes' activity. In order to perform these in vitro assays, the target $H$ pylori gene is transferred to Escherichia coli, where it is expressed, a recombinant $H$ pylori target protein is produced, and then the protein is isolated and purified.

Identification of inhibitory compounds is best accomplished by a process known as high throughput screening. This involves the automated microassay screening of the ability of large numbers of compounds $(>100000)$ to inhibit the activity of the target protein. Many companies have large libraries of compounds, including natural products, with which to screen. Confirmed "hits" are then tested for their ability to kill $H$ pylori.

Once a compound has been selected by virtue of its ability to inhibit the activity of a target protein and to kill $\mathrm{H}$ pylori, further research can be done to characterise the mechanism by which it inhibits, and by performing chemical structure activity relationship chemical synthesis studies to improve its ability to inhibit the target. Modern molecular modelling using computerised three dimensional techniques enable the target protein to be visualised in space. The inhibitory compound can also be incorporated into the system and oriented in the region of the protein where it exerts its inhibitory action. This enables the researcher to identify the properties of the compound that are particularly important for producing inhibition, and from that using various computer algorithms it is possible to introduce and visualise modifications that might enable the compound to be even more effective in inhibiting the action of the protein.

Once the candidate drug has been identified it enters the long period of trial in vitro, in vivo in animals and then in humans to confirm safety and efficacy.

\section{The genome and vaccine production}

The other area where the $H$ pylori genome is being used is in the development of vaccines. Vaccines have already been developed for $H$ pylori and are effective in mice, not only in preventing infection, but in eradicating infection already present. The vaccines produced so far have used $H$ pylori urease in association with an adjuvant such as cholera toxin. ${ }^{15}$ Without the adjuvant the vaccine does not work. To date the adjuvants used are too toxic for use in humans. Two problems therefore confront the researcher. The first is which antigens would be the best choice for the vaccine and second, how can the antigen be delivered in a way that enables the host to produce a suitable response.

Genome based vaccine research relies on the identification of genes which encode proteins which are exposed on the surface of $H$ pylori cells. It is these proteins which are positioned to interact with, and be targeted by the immune system. One class of proteins which is easy to identify are proteins incorporated into the outer membrane of the organism (the outer membrane proteins or OMPs). These proteins have characteristic structural motifs, making their genes easy to identify in the genome sequence. Some of the outer membrane proteins are exposed at the surface and are immunogenic, and so represent potential vaccine antigens. Among the outer membrane proteins which are immunogenic and have regions exposed to the surface are the porins. These are the proteins that provide the aqueous channels or pores that penetrate the lipopolysaccharide containing outer membrane. These pores are responsible for maintaining permeability, enabling the organism to metabolise and be in contact with the aqueous environment. There are a variety of porins, some responsible for large pores, others for small ones. Other surface proteins of importance are the adhesins. Considerable vaccine development work has already been undertaken using the urease enzyme, another protein identified by biochemical means some years ago.

Having identified a potential surface protein antigen, it is necessary to show that it is present and antigenically conserved in all strains of $H$ pylori. As is the case with drug targets, the $H$ pylori gene encoding the surface protein antigen is transferred to $E$ coli, where it is expressed, recombinant $H$ pylori antigen is produced, and the protein is isolated, purified and used to immunise mice and other animal models to evaluate its therapeutic potential. If the therapeutic trials are successful the antigen will subsequently need to be formulated into a vaccine and evaluated in humans.

\section{Other techniques}

An alternative method of identifying potential antigens for vaccines is to produce a vaccine from a sonicate of helicobacter in mice. Mouse serum containing antibody can be tested against a library of $H$ pylori antigens. ${ }^{16}$ This enables the researcher to identify which antigens produce antibodies in the mouse. Any one of these may then be produced, purified and tested for its effectiveness as a vaccine.

\section{Vaccine delivery}

The production of a suitable adjuvant is causing difficulty in that the most effective adjuvants are toxic. An alternative approach is to deliver the antigen to the host using another organism (the Trojan horse approach). Urease subunits A and B have been incorporated into attenuated Salmonella typhimurium which in turn has been given to mice. Nasal immunisation produced $67 \%$ protection $^{17}$ and oral immunisation $100 \%$ protection ${ }^{18}$ in two recent studies. This method avoids the necessity of using an adjuvant.

\section{Conclusion}

It will be some years before the products of genomic research become available in the pharmacy, but within the 12 years since the identification of $\mathrm{H}$ pylori we already have treatments that are $85-95 \%$ successful. There can be little doubt that within the next 10 years the fate of $H$ pylori will be sealed.

\footnotetext{
1 Lind T, Megraud F, Bardhan KD, et al. The MACH2 study: antimicrobial resistance in Helicobacter pylori therapy the impact of omeprazole. Gut 1997;41 (suppl 1):A89.
} 
2 Caselli M, Trevisani L, Tursi A, et al. Short-term low-dose triple therapy with azithromycin, metronidazole and lansoprazole appears highly effective for the eradication of Hel-

3 Labenz J, Stolte M, Peitz U, et al. One-week triple therapy with omeprazole, amoxycillin and either clarithromycin or metronidazole for cure of Helicobacter pylori infection. Aliment Pharmacol Ther 1996;10:207-10

4 Banatvala N, Davies GR, Abdi Y, et al. High prevalence of Helicobacter pylori metronidazole resistance in migrants to east London: relation with previous nitroimidazole exposure and gastroduodenal disease. Gut 1994;35:1562-6.

5 Moayyedi P, Sahay P, Tompkins DS, et al. Efficacy and optimum dose of omeprazole in a new 1-week triple therapy regimen to eradicate Helicobacter pylori. Eur f Gastroenterol Hepatol 1995;7:835-40.

6 Smith MA, Edwards DI. Redox potential and oxygen concentration as factors in the susceptibility of Helicobacter pylori to nitroheterocyclic drugs. F Antimicrob Chemother 1995;35:751-64.

7 Kasai K, Kobayashi R. The stomach spirochete occurring in mammals. I Parasitol 1919;6:1-10.

8 Phillips RH, Whitehead MW, Doig LA, et al. Eradication of $\mathrm{H}$ pylori using zinc based treatment [abstract]. Gut 1997;41(suppl 1):A100.

9 de Boer WA, Driessen WMM, Jansz AR, et al. Quadruple therapy compared with dual therapy for eradication of Helicobacter pylori in ulcer patients: results of a randomized prospective single-centre study. Eur $\mathcal{F}$ Gastroenterol Hepatol 1995; 7:1189-94.

10 Bardhan KD, Dallaire C, Eisold H, et al. Ranitidine bismuth citrate with clarithromycin for the treatment of duodenal ulcer. Gut 1997;41:181-6.
11 Savarino V, Mansi C, Mele MR, et al. A new 1-week therapy for Helicobacter pylori eradication: ranitidine bismuth citrate plus two antibiotics. Aliment Pharmacol Ther 1997;11: 699-703.

12 Williams MP, Hamilton MR, Sercombe JC, et al. Seven-day treatment for Helicobacter pylori infection: ranitidine bismuth citrate plus clarithromycin and tetracycline hydrochloride. Aliment Pharmacol Ther 1997;11:705-10.

13 Megraud F, Occhialini A, Bouchard S, et al. Nitazoxanide. A possible alternative to metronidazole in the regimens aiming to eradicate Helicobacter pylori [abstract]. Gut 1997;41(suppl 1):A100-1.

14 Tomb JF, White O, Kerlavage AR, et al. The complete genome sequence of the gastric pathogen Helicobacter pylori. Nature 1997;388:539-47.

15 Michetti P, Wadstrom T, Kraehenbuhl J-P, et al. Frontiers in Helicobacter pylori research: pathogenesis, host response, vaccine development and new therapeutic approaches. Eur f Gastroenterol Hepatol 1996;8:717-22.

16 Radcliff FJ, Doidge CV, Webb EA, et al. Success of a novel strategy: identification of new Helicobacter vaccine candidate antigens [abstract]. Gut 1997;41(suppl 1):A60.

17 Corthesy-Theulaz IE, Bachmann D, Hopkins S, et al. Mucosal immunization with attenuated salmonella expressing $\mathrm{H}$ pylori urease protect mice against Helicobacter infection [abstract]. Gut 1997; 41 (suppl 1):A59.

18 Gomez-Duarte OG, Yan ZX, Lucas B, et al. Urease subunits $\mathrm{A}$ and $\mathrm{B}$ delivered by attenuated Salmonella typhimurium vaccine strain protects mice against gastric colonization by Helicobacter pylori [abstract]. Gut 1997;41(suppl 1): A59-60. 